J. Lake Sci.(湖泊科学), 2019, 31(4): 961-968

DOI 10. 18307/2019. 0415

(c) 2019 by Journal of Lake Sciences

\title{
陆烧改性净水厂污泥对底泥内源磷释放的控制效果”
}

\author{
李书文, 周真明 ${ }^{* *}$, 杨思明, 刘淑坡, 李飞,苑宝玲 \\ (华侨大学土木工程学院, 厦门 361021)
}

\begin{abstract}
摘 要: 以自主研发的煆烧改性净水厂污泥 (C-WTPS) 作为污染底泥活性覆盖材料, 室内静态模拟实验研究 C-WTPS 覆 盖强度对控制底泥磷释放效果的影响, 分析 C-WTPS 和底泥中不同形态磷含量变化, 探讨 C-WTPS 薄层覆盖对上覆水体 中 $\mathrm{pH} 、 \mathrm{DO}$ 和 ORP 的影响. 结果表明, 实验历时 40 天, 在底泥 $\mathrm{TP}$ 释放强度为 $6.25 \sim 10.87 \mathrm{mg} /\left(\mathrm{m}^{2} \cdot \mathrm{d}\right)$ 时, 覆盖强度为 $0.25 、 0.50 、 1.00 、 1.50$ 和 $2.00 \mathrm{~kg} / \mathrm{m}^{2}$ 的 C-WTPS 对 TP 平均削减率分别为 $59.68 \% 、 75.71 \% 、 88.75 \% 、 92.42 \%$ 和 $96.28 \%$, 可见 覆盖强度为 $1.00 \mathrm{~kg} / \mathrm{m}^{2}$ 以上的 C-WTPS 能控制底泥中 $90 \%$ 以上 TP 释放. C-WTPS 吸附的磷主要以无机磷 (IP) 中的铁铝 结合态磷 (NAIP) 形式存在, 有机磷 (OP) 和䥻磷 (AP) 形式较少. C-WTPS 促进了底泥中易释放形态磷迁移到 C-WTPS 中, 并转化较为稳定的形态磷, 可见 C-WTP 覆盖不仅控制了底泥磷释放, 而且也削减了底泥磷释放风险. C-WTPS 覆盖后, 上 覆水体中 $\mathrm{pH}$ 开始呈现下降趋势, 最终维持在 $\mathrm{pH}=7$ 范围波动; C-WTPS 覆盖强度越大, 上覆水体 $\mathrm{pH}$ 下降也明显; C-WTPS 覆盖改善上覆水体中 DO 和 ORP 环境的效果不明显.
\end{abstract}

关键词: 底泥; 薄层覆盖;煅烧改性净水厂污泥; 磷释放; 不同形态磷

\section{The efficiency of controlling the phosphorus release from the sediment using calcined modified water purification plant sludge (C-WTPS)}

\author{
LI Shuwen, ZHOU Zhenming ** , YANG Siming, LIU Shupo, LI Fei \& YUAN Baoling \\ (College of Civil Engineering, Huaqiao University, Xiamen 361021, P.R.China)
}

\begin{abstract}
In this paper, the self-developed calcined water treatment plant sludge (C-WTPS) was used as an active sediment capping material for contaminated sediments. The static simulation experiment was conducted to study the effect of C-WTPS capping intensity on controlling phosphorus release from the sediment. Changes of phosphorus contents with different forms in the C-WTPS and sediment were analyzed, and the effect of thin-layer capping of C-WTPS on the pH, dissolved oxygen (DO) and oxidation-reduction potential (ORP) in overlying water was also discussed. The results showed that during a 40-day experiment, when the total phosphorus (TP) release intensity of the sediment was $6.25-10.87 \mathrm{mg} /\left(\mathrm{m}^{2} \cdot \mathrm{d}\right)$, the TP average reduction rate of C-WTPS with the capping intensity of $0.25,0.50,1.00,1.50$ and $2.00 \mathrm{~kg} / \mathrm{m}^{2}$ were $59.68 \%, 75.71 \%, 88.75 \%, 92.42 \%$ and $96.28 \%$, respectively. It means that C-WTPS with a capping intensity over $1.00 \mathrm{~kg} / \mathrm{m}^{2}$ could restrain over $90 \%$ TP from releasing. The phosphorus adsorbed by C-WTPS mainly exist in the form of iron-aluminum bounded phosphorus (NAIP) which were included in the inorganic phosphorus (IP), but the forms of organic phosphorus ( OP) and calcium phosphate ( AP) were less. C-WTPS promoted the migration of phosphorus which were easily released into the C-WTPS and turn it into the relatively stable phosphorus. It showed that the using capping material C-WTPS could not only control the release of phosphorus from the sediment but also reduce the potential risk of phosphorus release. During the process of C-WTPS capping, the $\mathrm{pH}$ of the overlying water began to decline at first, and maintained to be around 7 in the end. The higher intensity of capping was used, the lower $\mathrm{pH}$ in the overlying water was discovered. Although, the effect of improving the overlying water by using covering material C-WTPS in the DO and ORP were not obvious. Keywords: Sediment; thin-layer capping; calcined water treatment plant sludge; phosphorus release; different forms of phosphorus
\end{abstract}

* 福建省高校产学合作重大项目 (2018Y4101)、国家自然科学基金项目 (51678255,51878300)、福建省引导性项目 (2015Y0037)、泉州市高层次人才创新创业项目 (2017Z025)、华侨大学中青年教师科研提升资助计划项目 (ZQNPY313) 和华侨大学研究生科研创新基金项目联合资助. 2018-10-23 收稿; 2018-12-21 收修改稿. 李书文 (1991 ) ,男,硕士研究生; E-mail: 909635882@ qq.com.

** 通信作者; E-mail: zhenming@ hqu.edu.cn. 
城市水体富营养化修复是当今世界水污染防治的重要组成部分 ${ }^{[1]}$. 磷是水体富营养化的主要限制因子 之一, 控制水体磷浓度能有效抑制水体富营养化 ${ }^{[2]}$. 在外源磷得到有效控制条件下, 内源 (底泥) 磷是水体富 营养化的主要贡献者. 原位覆盖法 ${ }^{[3]}$ 能有效控制底泥磷释放, 是国内外学者研究热点. 污染底泥覆盖材料是 原位覆盖技术核心, 因此, 亟需研发货源广泛、经济有效、可规模化生产的底泥覆盖除磷材料. 目前底泥覆盖 除磷材料主要分为 4 大类: 铁盐、钙盐、镧改性和铝盐 ${ }^{[1]}$. 铁盐除磷材料主要有 Bephos ${ }^{\mathrm{TM}}{ }^{[4]}$, 但铁磷在还原条 件下容易再释放, 造成二次污染. 钻盐除磷材料主要有 $\mathrm{NCAP}^{[5]}$, 但钻磷在低 $\mathrm{pH}$ 或酸性条件下也易再释放. 镧改性的除磷材料主要有 phoslock ${ }^{\circledR[6-8]}$ 、镧改性磁铁矿 ${ }^{[9]}$ 和镧改性净水厂污泥 ${ }^{[10]}$ 等, 其中 phoslock ${ }^{\circledR}$ 在欧美 国家得到广泛工程应用 ${ }^{[6-8]}$, 但其形成的 $\mathrm{LaPO}_{4}$ 非常稳定, 能够永久除磷 ${ }^{[1]}$, 长时间大规模使用镧系除磷材料 可能会影响磷的自然循环, 甚至导致全球可用磷资源的枯竭. 铝盐除磷材料具有较好的磷吸附性能并且吸 附的磷不易再释放, 是具有较好应用前景的除磷材料 ${ }^{[1]}$.

净水厂污泥 (WTPS) 是净水厂在饮用水处理过程中加人铁盐、铝盐或铁铝盐絮凝沉淀水中悬浮物产生 的安全副产物 ${ }^{[11-12]}$. 相关研究表明, 非晶型结构的 Fe 和 Al 赋予了 WTPS 对较多污染物具有高吸附能力, 例 如磷 $(P)^{[13-14]}$ 、重金属 ${ }^{[15]}$ 、有机污染物 ${ }^{[16]}$ 和硫化氢 等 $^{[17]}$, 其中 WTPS 对磷的吸附研究深受研究者青睐. WTPS 具有高效稳定的磷吸附能力 ${ }^{[18-20]}$, 并且吸附的磷比较稳定且不易再释放 ${ }^{[21]}$. 但课题组前期研究表明, WTPS 直接使用过程中会向水体中释放氨氮和有机物 ${ }^{[22]}$, 造成二次污染, 因此, 课题组自主研发了煅烧改性 净水厂污泥 (C-WTPS), 不仅消除了向水体释放氨氮和有机物问题,而且还增加了磷吸附量 ${ }^{[23]}$.

本文以自主研发的 C-WTPS 作为污染底泥活性覆盖材料, 室内静态模拟实验研究 C-WTPS 覆盖强度对 控制底泥磷释放效果的影响, 分析 C-WTPS 和底泥中不同形态磷含量变化, 探讨 C-WTPS 薄层覆盖对上覆水 体中 $\mathrm{pH}$ 、溶解氧和氧化还原电位的影响. 研究成果可为 C-WTPS 覆盖控制底泥磷释放的实际应用提供理论 依据和技术支撑.

\section{1 材料与方法}

\section{1 实验材料}

实验使用的底泥取自华侨大学厦门校区白鹭湖表层底泥. 将所取表层底泥通过 16 目 (孔径 $1 \mathrm{~mm}$ ) 的篮 网、混合均匀得到实验底泥,其物理化学性质如表 1 所示. 实验所用的水为实验室自来水.

表 1 底泥物理化学性质

Tab.1 Physical and chemical properties of sediments

\begin{tabular}{ccccccccc}
\hline 指标 & $\mathrm{pH}$ & $\begin{array}{c}\mathrm{DO} / \\
(\mathrm{mg} / \mathrm{L})\end{array}$ & $\begin{array}{c}\mathrm{ORP} / \\
\mathrm{mV}\end{array}$ & $\begin{array}{c}\mathrm{TP} / \\
(\mathrm{mg} / \mathrm{kg})\end{array}$ & $\begin{array}{c}\mathrm{IP} / \\
(\mathrm{mg} / \mathrm{kg})\end{array}$ & $\begin{array}{c}\mathrm{OP} / \\
(\mathrm{mg} / \mathrm{kg})\end{array}$ & $\begin{array}{c}\mathrm{NAIP} / \\
(\mathrm{mg} / \mathrm{kg})\end{array}$ & $\begin{array}{c}\mathrm{AP} / \\
(\mathrm{mg} / \mathrm{kg})\end{array}$ \\
\hline 数值 & $6.75 \pm 0.09$ & $0.29 \pm 0.05$ & $-96 \pm 12$ & $3075.13 \pm 42.83$ & $2832.84 \pm 14.28$ & $276.63 \pm 7.14$ & $2187.92 \pm 19.99$ & $549.20 \pm 7.14$ \\
\hline
\end{tabular}

实验所用的 C-WTPS:WTPS 取自于厦门某净水厂的脱水后污泥,水厂絮凝剂以铝盐絮凝剂为主, 采集的 样品质地松软, 含水率约为 70\% 75\% , 自然风干后样品含水率降至 10\% 13\%. 将自然风干的样品放人马 弗炉中, 在 $400^{\circ} \mathrm{C}$ 下恒温炦烧 $2.5 \mathrm{~h}$ 制得 C-WTPS, 通过机械破碎笁分获得 $1 \sim 2 \mathrm{~mm}$ 的颗粒材料, C-WTPS 制 备参数选择详见参考文献 [23], 炦烧改性前后污泥理化性质变化详见参考文献 [24].

\section{2 实验装置与方法}

实验在容积为 $5 \mathrm{~L}$ 、直径 $150 \mathrm{~mm}$ 广口玻璃瓶中进行, 共有 12 个广口瓶, 分为 6 组, 每组 2 个平行样, 编 号为 $0^{\#} \sim 5^{\#}$, 其中, $0^{\#}$ 为对照系统 (未覆盖) $; 1^{\#} \sim 5^{\#}$ 为 C-WTPS 覆盖系统, C-WTPS 覆盖强度分别为 $0.25 、 0.50$ 、 $1.00 、 1.50$ 和 $2.00 \mathrm{~kg} / \mathrm{m}^{2}$. 每个瓶中底泥重量为 $1.21 \mathrm{~kg}$, 厚度约为 $5 \mathrm{~cm}$. 通过塑料软管利用虹吸原理将自来 水缓缓沿瓶壁加人瓶中, 上覆水体积为 $4 \mathrm{~L}$, 高度为 $20 \mathrm{~cm}$.

实验于 2018 年 3 月 7 日开始, 4 月 16 日结束, 历时 40 天; 玻璃瓶口敞开(不控制上覆水溶解氧 (DO) 浓 度), 放置在室内, 实验在室温下进行; 每天定时测定系统中水深 $10 \mathrm{~cm}$ 处水温、pH、DO 和氧化还原电位 $(\mathrm{ORP})$; 定期取 $50 \mathrm{ml}$ 水样, 分别测定水样中的总磷 ( TP)、正磷酸盐, 取水样后用自来水进行补充至原刻 
度线.

实验结束后收集各个系统底泥和 C-WTPS. 将收到到底泥混合均匀放人冰箱急冻 $2 \sim 3 \mathrm{~h}$, 再将其放人冷 冻干燥机干燥, 再碾细和过 100 目尼龙篮后待分析; 将收集的 C-WTPS 先用自来水清洗 3 次, 挑选出明显杂 质, 再用超纯水清洗 3 次, 放人 $105^{\circ} \mathrm{C}$ 的电烘箱干燥 $6 \mathrm{~h}$, 烘干的 C-WTPS 再碾细和过 100 目尼龙篎后待分析.

\section{3 项目测试方法}

1.3.1 水体相关指标测定方法 水温和 DO 采用 HQ30d 型便携式溶解氧测定仪测定 (HACH) ; pH 采用 STARTER3100 型便携式 $\mathrm{pH}$ 计 (上海奥豪斯仪器有限公司) 测定; ORP 采用 SX712 型便携式单参数 ORP 计 (上海三信仪器有限公司) 测定; $\mathrm{PO}_{4}^{3-}-\mathrm{P}$ 和 TP 浓度采用钿锑抗分光光度法 ${ }^{[25]}$ 测定.

1.3.2 底泥相关项目测定方法 采用 SMT 法 ${ }^{[26-27]}$ 测定底泥中不同形态磷的含量, 其中包括 TP、 IP (无机磷)、 $\mathrm{OP}$ (有机磷) 、 NAIP (以铁铝磷为主) 和 $\mathrm{AP}$ (以钙磷为主).

\section{4 数据处理}

上覆底泥污染物释放强度计算公式为:

$$
R_{n}=\left[V\left(C_{n}-C_{0}\right)+\sum_{i=1}^{n} V_{i}\left(C_{i}-C_{0}\right)\right] /(S \cdot t)
$$

式中, $n$ 为取样总次数; $R_{n}$ 为第 $n$ 次取样时底泥污染物释放强度 $\left(\mathrm{mg} /\left(\mathrm{m}^{2} \cdot \mathrm{d}\right)\right) ; V$ 为广口玻璃瓶中上覆水体 积 $(\mathrm{L}) ; C_{n}$ 为第 $n$ 次取样时上覆水中污染物浓度 $(\mathrm{mg} / \mathrm{L}) ; C_{0}$ 为初始污染物浓度 $(\mathrm{mg} / \mathrm{L}) ; i$ 为取样次数; $V_{i}$ 为 第 $i$ 次取水样量 $(\mathrm{L}) ; C_{i}$ 为第 $i$ 次取样时污染物浓度 $(\mathrm{mg} / \mathrm{L}) ; S$ 为广口玻璃瓶中底泥一水界面面积 $\left(\mathrm{m}^{2}\right) ; t$ 为 污染物释放时间 (d).

上覆水磷削减率 $(P)$ 计算公式为:

$$
P=\left(C_{\mathrm{NC} i}-C_{\mathrm{C} i}\right) / C_{\mathrm{NC} i} \times 100 \%
$$

式中, $C_{\mathrm{C} i}$ 为取样时覆盖系统上覆水磷浓度 $(\mathrm{mg} / \mathrm{L}), C_{\mathrm{NC} i}$ 为取样时空白系统上覆水磷浓度 $(\mathrm{mg} / \mathrm{L}), i$ 为取样 次数.

采用 Origin 8.5 软件通过方差分析研究覆盖系统与空白系统之间削磷污染物效果的差异.

\section{2 结果与讨论}

\section{1 不同覆盖强度的 C-WTPS 控制底泥磷释放效果}

在历时 $40 \mathrm{~d}$ 静态模拟实验中, 各个系统上覆水温度为 $18.1 \sim 26.3^{\circ} \mathrm{C}$; 各系统中上覆水中 $\mathrm{PO}_{4}^{3-}-\mathrm{P}$ 和 $\mathrm{TP}$ 浓 度变化见图 1. 在对照系统上覆水中, $\mathrm{PO}_{4}^{3-}-\mathrm{P}$ 占 $\mathrm{TP}$ 的 $74.82 \% \sim 99.15 \%$, 均值为 $94.42 \%$, 表明白路湖底泥释 放的磷以正磷酸盐为主; 底泥 TP 的释放强度为 $6.25 \sim 10.87 \mathrm{mg} /\left(\mathrm{m}^{2} \cdot \mathrm{d}\right)$, 均值为 $8.90 \mathrm{mg} /\left(\mathrm{m}^{2} \cdot \mathrm{d}\right)$. 与对照相 比, 覆盖强度为 $0.25 、 0.50 、 1.00 、 1.50$ 和 $2.00 \mathrm{~kg} / \mathrm{m}^{2}$ 的 C-WTPS 对 $\mathrm{PO}_{4}^{3-}-\mathrm{P}$ 平均削减率分别为 $60.57 \%$ 、 $76.91 \% 、 90.89 \% 、 94.17 \%$ 和 $97.51 \%$, 对 TP 平均削减率分别为 $59.68 \% 、 75.71 \% 、 88.75 \% 、 92.42 \%$ 和 $96.28 \%$; 方差分析表明, 覆盖强度为 $0.25 \mathrm{~kg} / \mathrm{m}^{2}$ 的 C-WTPS 覆盖系统与对照系统之间削减 TP 效果无明显差异 $(P>$ $0.05)$, 其他覆盖强度系统与对照系统均有显著性差异 $(P<0.05)$; 可见覆盖强度为 $1 \mathrm{~kg} / \mathrm{m}^{2}$ 以上的 C-WTPS 能控制底泥中 $90 \%$ 以上 TP 释放, 主要是因为 C-WTPS 含有较丰富的铁铝非晶结构在材料中形成的活性吸 附位点通过配体交换、表面沉淀或络合和微孔扩散等 ${ }^{[28-31]}$ 方式将磷固定在材料中, 从而达到除磷目的 (图 1).

C-WTPS 覆盖系统 TP 削减率与 C-WTPS 覆盖强度之间的关系如图 2 所示, 得到拟合关系式为: $y=$ $-15.616 x^{2}+53.9887 x+49.4992\left(R^{2}=0.9415\right)$, 覆盖强度 $1.73 \mathrm{~kg} / \mathrm{m}^{2}$ 的 C-WTPS 覆盖系统 TP 削减率最大理论 值为 $96.20 \%$, 与覆盖强度 $2.00 \mathrm{~kg} / \mathrm{m}^{2}$ 的 TP 削减率实验值相近.

\section{2 各系统 C-WTPS 和底泥中不同形态磷含量变化}

2.2.1 各系统 C-WTPS 中不同形态磷含量变化 在历时 $40 \mathrm{~d}$ 静态模拟实验中,各覆盖系统 C-WTPS 中不同形 态磷含量变化见图 3. 实验后, C-WTPS 中 TP、IP 和 NAIP 含量显著性增加 $(P<0.05)$; 随着 C-WTPS 覆盖强度 的增加, C-WTPS 中 TP、IP 和 NAIP 含量均减小; 主要原因底泥磷释放强度一定的情况下, C-WTPS 覆盖强度 越大, 材料提供的磷吸附位点越多, 可能会出现吸附位点未被充分利用, 致使 C-WTPS 磷吸附量随着覆盖强 

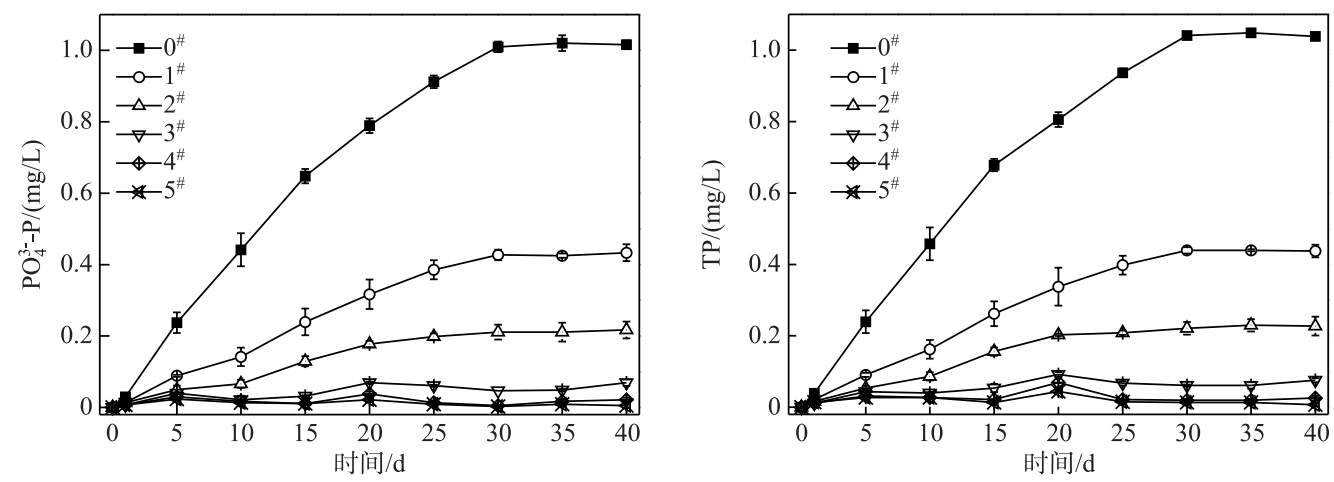

图 1 各系统上覆水中 $\mathrm{PO}_{4}^{3-}-\mathrm{P}$ 和 $\mathrm{TP}$ 浓度的变化

Fig. 1 Changes of $\mathrm{PO}_{4}^{3-}-\mathrm{P}$ and TP concentrations of overlying water in each system

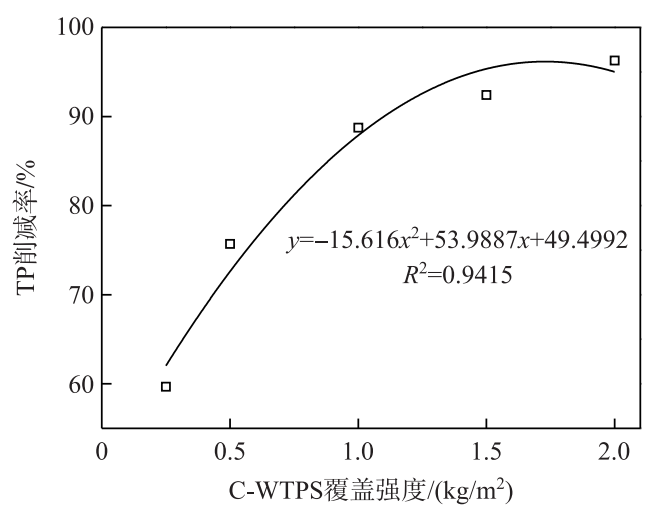

图 2 C-WTPS 覆盖强度与 TP 削减率的关系

Fig.2 Relationship between TP reduction rate and C-WTPS capping strength

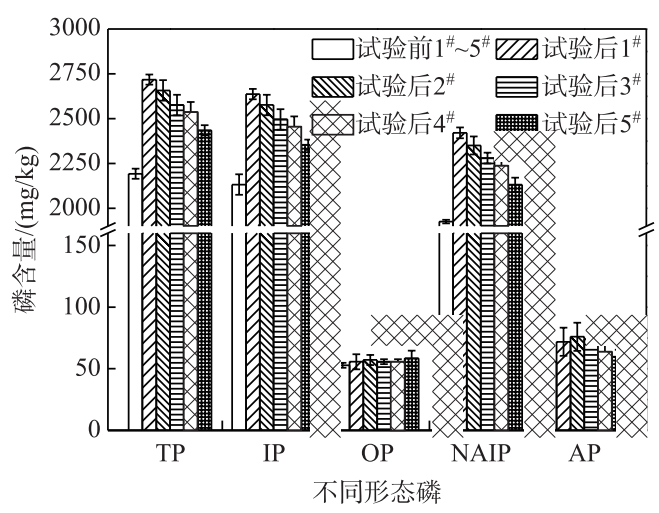

图 3 各覆盖系统 C-WTPS 中 不同形态磷含量的变化

Fig.3 Changes of phosphorus content in different forms of C-WTPS in each system
度的增大而减小. 这与课题组前期研究结果一致 ${ }^{[32]}$. 实 验后, 各覆盖系统 C-WTPS 中的 TP 平均含量分别增加 了 524.97、464.39、383.63、343.25 和 $242.29 \mathrm{mg} / \mathrm{kg}$; 无机 磷 (IP) 平均含量分别增加了 504.78、444.20、363.44、 323.06 和 $228.10 \mathrm{mg} / \mathrm{kg}$; 有机磷 (OP) 平均含量增加不 明显; 铁铝结合态磷 (NAIP) 平均含量分别增加了 $494.68 、 424.01 、 353.34 、 310.94$ 和 $204.94 \mathrm{mg} / \mathrm{kg}$; 钻磷 (AP) 平均含量分别增加了 $16.15 、 20.19 、 18.17 、 8.08$ 和 $4.04 \mathrm{mg} / \mathrm{kg}$; C-WTPS 吸附的磷主要以 IP 中的 NAIP 形 式存在, OP 和 AP 形式较少 ${ }^{[33]}$, 与课题组前期研究结果 一致 ${ }^{[23-24]}$. 各覆盖系统中 C-WTPS 磷吸附量分别为 $0.53 、 0.46 、 0.38 、 0.34$ 和 $0.24 \mathrm{mg} / \mathrm{g}$; 磷初始浓度为 $2 \mathrm{mg} / \mathrm{L}$ 时, C-WTPS 的最大吸附量为 $2.95 \mathrm{mg} / \mathrm{g}^{[32]}$, C-WTPS 利用效率较低, 主要是由于覆盖系统上覆水体 中磷浓度较低 (均小于 $0.5 \mathrm{mg} / \mathrm{L}$ ), 致使 C-WTPS 磷吸 附量较低, 可见 C-WTPS 在实际使用时, 需要结合实际 水体磷初试浓度和 C-WTPS 覆盖强度估算 C-WTPS 磷 削减量, 以免高估 C-WTPS 除磷效果, 致使难达到预期 目的.

2.2 .2 各系统底泥中不同形态磷含量变化 实验前, 底 泥中 TP、IP、OP、NAIP 和 AP 平均含量分别为 3075.13、 $2832.84 、 276.63 、 2187.92$ 和 $549.20 \mathrm{mg} / \mathrm{kg}$, 其中 TP 中的 IP 和 NAIP 分别占 $92.12 \%$ 和 $71.15 \%$, 说明白鹭湖底泥 中磷主要以 NAIP 为主要无机磷形态存在, 底泥释放磷 的风险较大. 实验后, 对照系统底泥中 TP、IP、OP、NAIP 和 AP 平均含量分别减少了 $111.05 、 100.95 、 20.19 、 84.80$ 和 $-25.24 \mathrm{mg} / \mathrm{kg}$. 实验后, 各覆盖系统底泥 TP 含量分别 减少了 $111.05 、 151.43 、 181.72 、 222.10$ 和 $282.68 \mathrm{mg} / \mathrm{kg}$, 分别占试验前底泥 TP 的 3.61\%、4.92\%、5.91\%、7.22\% 和 $9.20 \%$, 底泥磷的迁移转化量为 $111.05 \sim 282.68$ $\mathrm{mg} / \mathrm{kg}$, 底泥磷的迁移转化量与 C-WTPS 覆盖强度呈正 
相关 (图 4). 说明 C-WTPS 促进了底泥中易释放形态磷迁移到 C-WTPS 中, 并转化较为稳定的形态磷, 可见 C-WTP 覆盖不仅控制了底泥磷释放, 而且也削减了底泥磷释放风险.

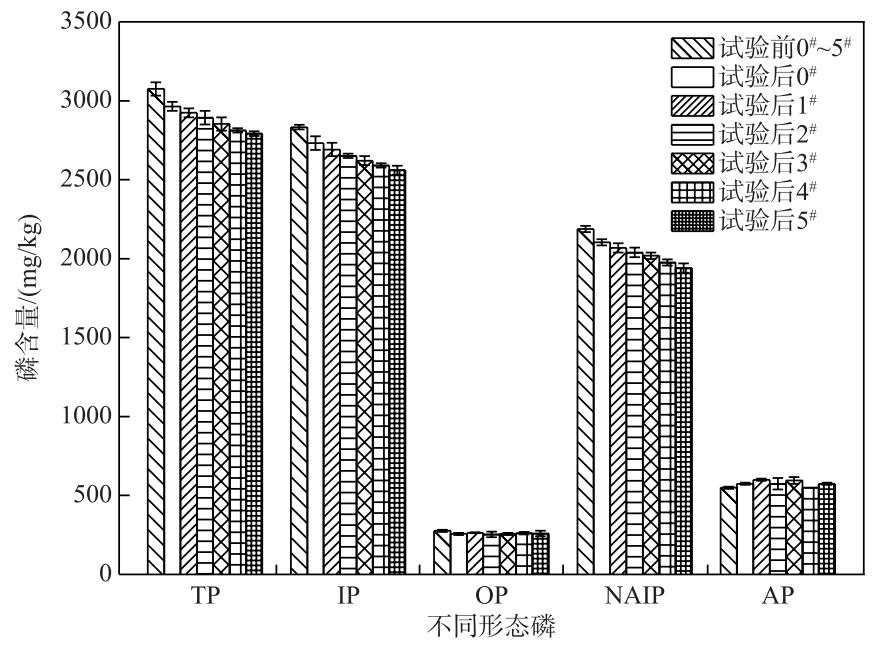

图 4 各系统底泥中不同形态磷含量的变化

Fig.4 Changes of phosphorus content in different forms of sediment in each system

\subsection{C-WTPS 覆盖对上覆水环境因子的影响}

$2.3 .1 \mathrm{pH}$ 在历时 $40 \mathrm{~d}$ 静态模拟实验中, 各系统上覆水中 $\mathrm{pH}$ 的变化如图 5 所示. C-WTPS 覆盖系统中前 $15 \mathrm{~d}$ 期间 $\mathrm{pH}$ 呈现下降趋势, 其主要原因是由于浓度差的关系, 底泥向上覆水释放各类阴阳离子(如金属离 子、氮磷营养盐等) ; 上覆水从好氧向缺氧状态转变, ORP 的降低等因素改变了上覆水中铁盐、硫酸盐等的种 类和浓度, 导致上覆水 $\mathrm{pH}$ 的下降 ${ }^{[24,34-36]}$. 实验 $15 \mathrm{~d}$ 后, 方差分析表明, 对照系统和各覆盖系统的 $\mathrm{pH}$ 均存在 显著性差异 $(P<0.05)$, 并且 C-WTPS 覆盖强度越大 $\mathrm{pH}$ 差异越显著. 说明 C-WTPS 能够改变水体 $\mathrm{pH}$, 且当上 覆水显碱性时, $\mathrm{pH}$ 会下降; 当上覆水显酸性时, $\mathrm{pH}$ 会上升, 但始终在 $\mathrm{pH}=7$ 小范围内波动. 这与课题组前期 研究结果一致 ${ }^{[24]}$. 其原因有, 首先是 C-WTPS 中含有大量的无定型铝铁化合物, C-WTPS 吸附磷的方式为表 面络合、配体 (离子) 交换、表面沉淀等方式可能改变了上覆水阴阳离子 (如 $\mathrm{H}^{+}$和金属离子) 的种类和浓度.

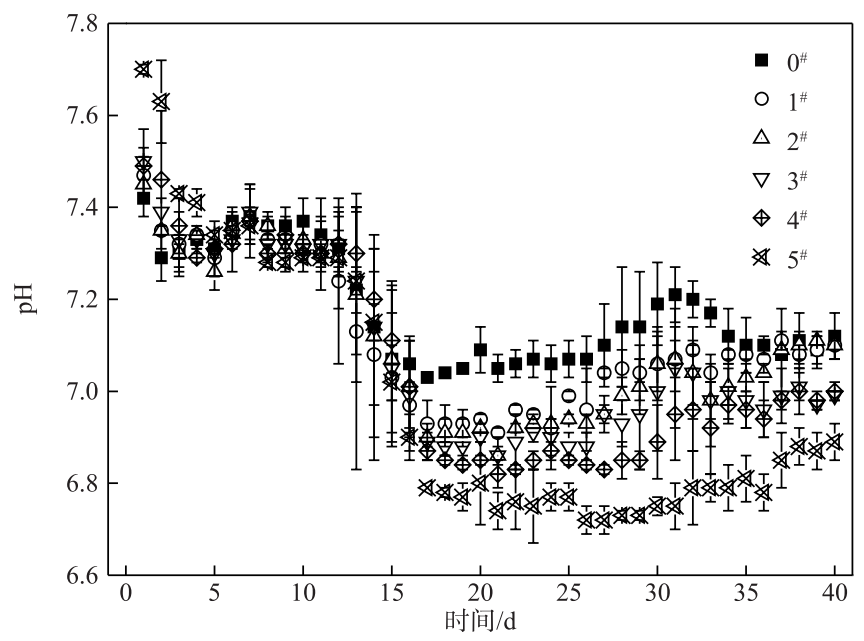

图 5 各系统上覆水中 $\mathrm{pH}$ 变化

Fig.5 Changes of $\mathrm{pH}$ of overlying water in each system 
Yang 等 ${ }^{[31]}$ 研究表明 C-WTPS 在配体 (离子) 交换过程会释放 $\mathrm{H}^{+}$、TOC、金属离子和其他阴离子, 释放的 $\mathrm{H}^{+}$和 金属离子水解产生的 $\mathrm{H}^{+}$导致了上覆水 $\mathrm{pH}$ 的减小; 其次是上覆水溶解氧小于 $1 \mathrm{mg} / \mathrm{L}$ 处于缺氧状态下, 微生 物分解有机物可能产生有机酸, 导致 $\mathrm{pH}$ 下降 ${ }^{[36]}$.

2.3.2 DO 和 ORP 在历时 $40 \mathrm{~d}$ 静态模拟实验中, 各系统上覆水中 DO 和 ORP 的变化如图 6 所示.
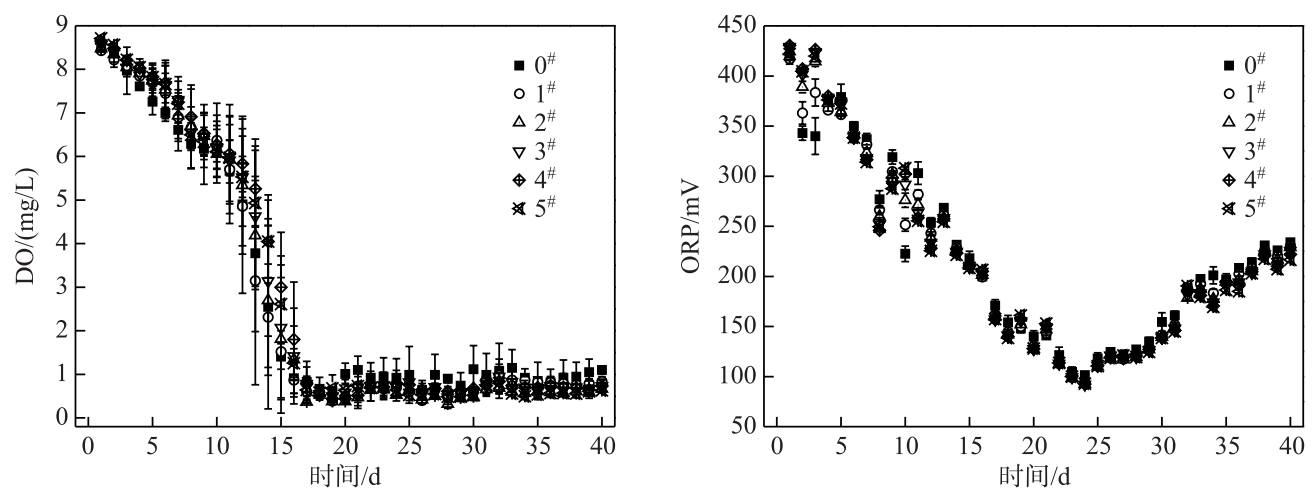

图 6 各系统上覆水中 DO 浓度和 ORP 的变化

Fig.6 Changes of DO concentrations and ORP of overlying water in each system

各系统上覆水体中的 DO 浓度均呈现先降低后保持平稳的趋势; 在前 $15 \mathrm{~d}$ 各系统从好氧状态逐渐变成缺 氧状态, 实验 $15 \mathrm{~d}$ 后各系统均为缺氧状态 $(\mathrm{DO}<1 \mathrm{mg} / \mathrm{L})$, 方差分析表明, C-WTPS 覆盖系统上覆水中 DO 浓度 与对照系统没有显著性差异 $(P>0.05)$; 可见各系统耗氧速率大于空气复氧速率, 说明 $\mathrm{C}-\mathrm{WTPS}$ 覆盖控制底 泥有机物释放效果不明显,从而改善上覆水体 DO 环境的效果不明显 (图 6).

各系统上覆水中的 ORP 均呈现先降低后增加的趋势 (图 6). 在 $24 \mathrm{~d}$ 时达到最小值为 $97 \mathrm{mV}$, 其原因可 能是 DO 和 $\mathrm{pH}$ 降低逐渐使还原态与氧化态物质浓度比值增大导致 ORP 减小; 在 $24 \mathrm{~d}$ 后 DO 浓度趋于稳定, 系统中的还原态物质开始逐渐减少导致 ORP 增加; 此外还有离子浓度和温度等因素也会间接影响 $\mathrm{ORP}^{[37]}$. 方差分析表明, C-WTPS 覆盖系统上覆水中 ORP 与对照系统没有显著性差异 $(P>0.05)$, 说明 C-WTPS 覆盖 改善上覆水中 ORP 环境的效果不明显.

\section{3 结论}

1) 覆盖强度为 $0.25 、 0.50 、 1.00 、 1.50$ 和 $2.00 \mathrm{~kg} / \mathrm{m}^{2}$ 的 C-WTPS 对 $\mathrm{PO}_{4}^{3-}-\mathrm{P}$ 平均削减率分别为 $60.57 \%$ 、 $76.91 \% 、 90.89 \% 、 94.17 \%$ 和 $97.51 \%$, 对 TP 平均削减率分别为 $59.68 \% 、 75.71 \% 、 88.75 \% 、 92.42 \%$ 和 $96.28 \%$; 可见覆盖强度为 $1.00 \mathrm{~kg} / \mathrm{m}^{2}$ 以上的 C-WTPS 能控制底泥中 $90 \%$ 以上 TP 的释放.

2) C-WTPS 吸附的磷主要以无机磷中的铁铝结合态磷形式存在, C-WTPS 促进了底泥中易释放形态磷 迁移到 C-WTPS 中, 并转化较为稳定的形态磷.

3) C-WTPS 覆盖后, 上覆水体中 $\mathrm{pH}$ 开始呈现下降趋势, 最终维持在 $\mathrm{pH}=7$ 范围波动; C-WTPS 覆盖改善 上覆水体中 DO 和 ORP 环境的效果不明显.

\section{4 参考文献}

[ 1 ] Lürling M, Mackay E, Reitzel K et al. Editorial-A critical perspective on geo-engineering for eutrophication management in lakes. Water Research, 2016, 97: 1-10.

[ 2 ] Lewis WM, Wurtsbaugh WA, Paerl HW. Rationale for control of anthropogenic nitrogen and phosphorus to reduce eutrophication of inland waters. Environmental Science \& Technology, 2011, 45(24) : 10300-10305.

[ 3 ] Spears BM, Maberly SC, Pan G et al. Geo-engineering in lakes: a crisis of confidence? Environmental Science \& Technology, 2014, 48: 9977-9979.

[ 4 ] Zamparas M, Drosos M, Georgiou Y et al. A novel bentonite-humic acid composite material Bephos ${ }^{\mathrm{TM}}$ for removal of phos- 
phate and ammonium from eutrophic waters. Chemical Engineering Journal, 2013, 225: 43-51.

[ 5 ] Ding SM, Chen MS, Cui JZ et al. Reactivation of phosphorus in sediments after calcium-rich mineral capping: Implication for revising the laboratory testing scheme for immobilization efficiency. Chemical Engineering Journal, 2018, 331: 720-728.

[ 6 ] Douglas GB, Lurling M, Spears BM. Assessment of changes in potential nutrient limitation in an impounded river after application of lanthanum-modified bentonite. Water Research, 2016, 97: 47-54.

[ 7 ] Waajen G, Van OF, Douglas G et al. Management of eutrophication in Lake De Kuil (The Netherlands) using combined flocculant-Lanthanum modified bentonite treatment. Water Research, 2016, 97: 83-95.

[ 8 ] Ding SM, Sun Q, Chen X et al. Synergistic adsorption of phosphorus by iron in lanthanum modified bentonite (Phoslock) : New insight into sediment phosphorus immobilization. Water Research, 2018, 134: 32-43.

[ 9 ] Fang L, Liu R, Li J et al. Magnetite/Lanthanum hydroxide for phosphate sequestration and recovery from lake and the attenuation effects of sediment particles. Water Research, 2018, 130: 243-254.

[10] Wang C, Wu Y, Wang Y et al. Lanthanum-modified drinking water treatment residue for initial rapid and long-term equilibrium phosphorus immobilization to control eutrophication. Water Research, 2018, 137: 173-183.

[11] Ippolito JA, Barbarick KA, Elliott HA. Drinking water treatment residuals: a review of recent uses. Journal of Environmental Quality, 2011, 40: 1-12.

[12] Okuda T, Nishijima W, Sugimoto M et al. Removal of coagulant aluminum from water treatment residuals by acid. Water Research, 2014, 60: 75-81.

[13] Li Z, Jiang N, Wu F et al. Experimental investigation of phosphorus adsorption capacity of the waterworks sludges from five cities in China. Ecological Engineering, 2013, 53: 165-172.

[14] Wang C, Liang J, Pei Y et al. A method for determining the treatment dosage of drinking water treatment residuals for effective phosphorus immobilization in sediments. Ecological Engineering, 2013, 60(11) : 421-427.

[15] Lin L, Xu X, Papelis C et al. Innovative use of drinking water treatment solids for heavy metals removal from desalination concentrate: synergistic effect of salts and natural organic matter. Chemical Engineering Research \& Design, 2017,120 : 231-239.

[16] Punamiya P, Sarkar D, Rakshit S et al. Effectiveness of aluminum-based drinking water treatment residuals as a novel sorbent to remove tetracyclines from aqueous medium. Journal of Environmental Quality, 2013, 42 : 1449-1459.

[17] Wang CH, Pei YS. The removal of hydrogen sulfide in solution by ferric and alum water treatment residuals. Chemosphere, 2012, 88: 1178-1183.

[18] Agyin-Birikorang S, O'Connor GA. Lability of drinking water treatment residuals (WTR) immobilized phosphorus: aging and pH effects. Journal of Environmental Quality, 2007, 36: 1076-1085.

[19] Oliver IW, Grant CD, Murray RS. Assessing effects of aerobic and anaerobic conditions on phosphorus sorption and retention capacity of water treatment residuals. Journal of Environmental Management, 2011, 92: 960-966.

[20] Wang CH, Wang ZY, Lin L et al. Effect of low molecular weight organic acids on phosphorus adsorption by ferric-alum water treatment residuals. Journal of Hazardous Materials, 2012, 203/204: 145-150.

[21] Wang CH, Fei CB, Pei YS. Stability of P saturated water treatment residuals under different levels of dissolved oxygen. Clean Soil Air Water, 2012, 40: 844-849.

[22] Huang HS, Yang ZM, Zhou ZM et al. Efficiency of controlling nitrogen and phosphorus release from sediment using thinlayer capping with water treatment plant sludge. Journal of Huaqiao University: Natural Science, 2016, 37(3) : 347-351. [黄华山, 杨志敏, 周真明等. 净水厂污泥覆盖控制底泥氮磷释放效果. 华侨大学学报: 自然版, 2016, 37(3): 347-351.]

[23] Liu QD, Zhou ZM, Zhang HZ et al. Parameter optimization of preparing phosphorus removal material by using calcined water treatment plant sludge. Journal of Huaqiao University: Natural Science, 2018, 39(1): 51-56. [刘啟迪, 周真明, 张 红忠等. 煅烧改性净水厂污泥制备除磷材料工艺参数优化. 华侨大学学报: 自然科学版, 2018, 39(1): 51-56.]

[24] Zhou Z, Liu Q, Li S et al. Characterizing the correlation between dephosphorization and solution $\mathrm{pH}$ in a calcined water treatment plant sludge. Environmental Science \& Pollution Research, 2018, 25(19) : 18510-18518.

[25] Ministry of Environmental Protection of the People's Republic of China, Editorial Board of Water and Wastewater Monitoring and Analysis Methods ed. Water and wastewater monitoring and analysis methods: 4th edition. Beijing: China Envi- 
ronmental Science Press, 2002. [ 国家环境保护总局《水和废水监测分析方法》编委会. 水和废水监测分析方法: 第 4 版. 北京: 中国环境科学出版社, 2002.]

[26] Ruban V, Lópezsánchez JF, Pardo P et al. Selection and evaluation of sequential extraction procedures for the determination of phosphorus forms in lake sediment. Journal of Environmental Monitoring Jem, 1999, 1(1) : 51-56.

[27] González Medeiros JJ, Pérez CB, Fernández GE. Analytical phosphorus fractionation in sewage sludge and sediment samples. Analytical and Bioanalytical Chemistry, 2005, 381(4) : 873-878.

[28] Ippolito JA, Barbarick KA, Heil DM et al. Phosphorus retention mechanisms of a water treatment residual. Journal of Environmental Quality, 2003, 32(5): 1857-1864.

[29] Makris KC. Long-term stability of sorbed phosphorus by drinking-water treatment residuals: mechanisms and implications [Dissertation]. Florida: University of Florida, 2004.

[30] Al-Tahmazi T, Babatunde AO. Mechanistic study of P retention by dewatered waterworks sludges. Environmental Technology \& Innovation, 2016, 6: 38-48.

[31] Yang Y, Zhao YQ, Babatunde AO et al. Characteristics and mechanisms of phosphate adsorption on dewatered alum sludge. Separation \& Purification Technology, 2006, 51(2) : 193-200.

[32] Liu QD. Study on preparation of water environmental phosphorus removal materials by calcining water treatment plant sludge[Dissertation]. Xiamen: Huaqiao University, 2018. [ 刘啟迪. 净水厂污泥煅烧制备水环境除磷材料 [ 学位论 文]. 厦门: 华侨大学, 2018.]

[33] Hou QJ, Meng PP, Pei HY et al. Phosphorus adsorption characteristics of alum sludge: adsorption capacity and the forms of phosphorus retained in alum sludge. Materials Letters, 2018, 229: 31-35.

[34] Wu YH, Wen YJ, Zhou JX et al. Phosphorus release from lake sediments: effects of $\mathrm{pH}$, temperature and dissolved oxygen. KSCE Journal of Civil Engineering, 2014, 18(1): 323-329.

[35] Li ZR, Sheng YQ, Yang J et al. Phosphorus release from coastal sediments: Impacts of the oxidation-reduction potential and sulfide. Marine Pollution Bulletin, 2016, 113(1/2): 176-181.

[36] Huang TL, Zhou RY, Xia C et al. Effects of oxidation-reduction potential and microorganism on the release of phosphorus from sediments. Environmental Chemistry, 2014, 33(6): 930-936. [ 黄廷林, 周瑞媛, 夏超等. 氧化还原电位及微生物 对水库底泥释磷的影响. 环境化学, 2014, 33(6): 930-936.]

[37] Xiang J, Xu LP, Li HP et al. Research on and application of oxidation-reduction potential. Earth and Environment, 2014, 42(3) : 430-436. [ 向交, 徐丽萍, 李和平等. 氧化还原电位的研究及应用. 地球与环境, 2014, 42(3): 430-436.] 